\title{
Designer Interfaces for Label-free Biosensing
}

\author{
Tatsuro Goda ${ }^{1,2 *}$ \\ ${ }^{1}$ Department of Biomedical Engineering, Faculty of Science and Engineering, Toyo University, \\ 2100 Kujirai, Kawagoe, Saitama 350-8585, Japan \\ ${ }^{2}$ Nano Innovation Institute, Inner Mongolia University for Nationalities, \\ No. 22 HuoLinHe Street, Tongliao, Inner Mongolia 028000, China
}

(Received August 26, 2020; accepted October 20, 2020)

Keywords: aptamer, molecular switch, cell membrane mimicry, biomarker, influenza virus

Nowadays, there is huge demand for biosensing in point-of-care and wearable formats. Label-free biosensors meet the criteria because they are fast and straightforward without the need for labeling to detect target molecules. Instead, they have to detect a small amount of analyte among irrelevant molecules with a high signal-to-noise ratio by performing efficient biorecognition. Therefore, it is crucial to design and control the structure of the selective layer at the interface between the sample solution and the transducer. The selective layer contains capturing elements from natural or synthetic origins. Artificial receptors are a new class of components with improved stability, durability, and quality as compared with natural antibodies and enzymes. This review addresses the functionalization of the selective layer. It possesses smart, biomimetic, and non-fouling characteristics using synthetic receptors and molecules. Molecular switches made of an oligonucleotide can respond to the target upon environmental stimuli. Their responsiveness is tunable by designing the probe sequence. Cell membranes in which bioreceptors are embedded in self-assembled phospholipid bilayer membranes provide hints for developing an advanced biosensing platform. Cell membrane-mimetic surfaces are robust through covalent contacts and resist nonspecific adsorption. The adaptation of a cell surface receptor efficiently improves the binding specificity. These technologies are essential for developing next-generation label-free biosensors.

\section{Introduction}

Biosensors are used to analyze the type and concentration of a chemical or biological component of interest in a body fluid such as blood, saliva, or urine. A certain biomarker molecule indicates the onset of a specific disease. ${ }^{(1)}$ Early diagnosis through biomarker detection is essential to cure cancer or infection. Therefore, sensitivity and selectivity in recognizing a target molecule are essential requirements for biosensors. Biosensors are mainly composed of a transducer (detector) and a selective layer (Fig. 1). The transducer converts recognition events into measurable quantities. The selective layer is placed between the transducer and the sample solution to discriminate the target analyte from impurities. Most biosensors are used in

*Corresponding author: e-mail: goda@toyo.jp

https://doi.org/10.18494/SAM.2021.3065 


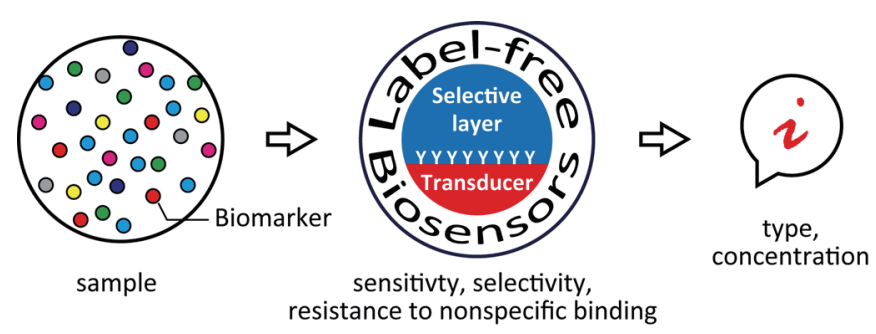

Fig. 1. (Color online) A label-free biosensor determines the type and concentration of a target biomolecule without labeling. It comprises a selective layer and a transducer. This review highlights the development of functional selective layers with smart, biomimetic, and non-fouling characteristics for advanced label-free biosensing.

combination with preliminary separation or labeling techniques for the selective detection of an analyte. Possible labels include enzymes, antibodies, nucleic acids, fluorescence, radioisotopes, nanocolloids, and microbeads. These tags can report the number of a target molecule to the transducer or even amplify the signal. As a result, an extremely small amount of bioanalyte can be identified. Although it is a standard method, label-based biosensing is tedious, expensive, and time-consuming. Moreover, the introduction of labels modulates the original properties of the target analyte such as the mass, charge, dielectric constants, and conformation, occasionally leading to adverse effects on the affinity-based biorecognition system. ${ }^{(2)}$

Label-free biosensing is another strategy for detecting an analyte. It requires no labeling processes because the transducer can convert the intrinsic characteristics of the analyte, including the mass, volume, charge, permittivity, autofluorescence, light scattering, and refractive index, into a signal. ${ }^{(3)}$ Therefore, it has advantages of simplicity, cost-efficiency, and rapidity. These features open up applications for point-of-care testing and wearable monitoring. (4) Owing to the absence of labels, the transduction mechanisms should be sensitive enough to detect a tiny amount of the target. Moreover, the selective layer plays an essential role in acquiring the target-specific signal. It has to accumulate the analyte from sample solutions by the affinity between the surface-immobilized receptor and the free target. ${ }^{(5)}$ This is because any molecules that reside on a sensing area, regardless of whether the binding is specific or nonspecific, can generate a signal. Impurities and miscellaneous molecules should be repelled from the selective layer to reduce the background signal or noise. Nonspecific binding inevitably occurs by intermolecular forces such as van der Waals forces, electrostatic forces, and hydrogen bonding. Traditionally, a hydrophilic supporting layer has been used to reduce nonspecific adsorption on a label-free biosensor. ${ }^{(6)}$ However, almost complete removal of nonspecifically adsorbed molecules is necessary for the label-free biosensing of practical and clinical samples.

Label-free biosensing has made significant progress in the last few decades owing to the advances in both the transducer and the selective layer. This paper summarizes the concept and practice of advanced label-free biosensing, emphasizing the development of the functional selective layer achieved by the author and coworkers. The original concepts comprise three elements: (i) smart binding using stimuli-responsive ligands, (ii) biomembrane-mimicking affinity interactions, and (iii) non-fouling surfaces produced by zwitterions. Concept (i) enables the development of functionalized label-free biosensing based on the stimuli responsiveness. The functional layers are made of macromolecular and supermolecular assemblies that can 
generate a signal through the conformational transitions upon molecular recognition. Molecularswitch-based signal transduction will help us to acquire sophisticated responses such as logic gate and systemic functions. ${ }^{(7)}$ The use of artificial ligands provides molecular robustness and designability compared with biomolecular ones such as enzymes and antibodies (Table 1). Concepts (ii) and (iii) are biomimetic approaches. Biomimicry is a strategy that empowers engineering by adapting complete biosystems that living organisms have acquired through trial and error during the course of evolution. A series of affinity binding reactions on the cell surface spurs us to explore efficient biorecognition and signal transduction systems. At the same time, inhibiting the nonspecific adsorption of irrelevant biomolecules on the cell surface underpins the binding specificity and selectivity. The anti-fouling properties are produced by a unique hydration state of the phospholipid headgroup aligned on the cell membranes. ${ }^{(8,9)}$ The biomimicry design of an anti-fouling agent on the biosensor surface is essential for sensitive and selective biosensing without labeling. The above topics are introduced and discussed in the following sections.

\section{DNA Aptamers for Biomarker Detection}

DNA aptamers serve as receptors for a wide variety of targets, including ions, nutrients, and proteins, because of the freedom of sequences and tertiary structures. ${ }^{(10)}$ A DNA aptamer exhibits a high binding affinity and selectivity to the target. Compared with RNA, DNA has high endurance against enzymatic digestion in biological samples. DNA can be chemically modified to add anchor or linker molecules for surface immobilization. Motivated by these features, anti-thrombin DNA aptamer with a 6-mercaptohexyl group in the 5' end of the oligonucleotide was chemically immobilized on a gold electrode as an extended gate of a fieldeffect transistor (FET) (Fig. 2). ${ }^{(1)}$ The aptamer density was determined by chronocoulometry. Thrombin is a major blood coagulant factor and an ideal biomarker for pulmonary metastases and atherosclerosis. FET biosensors can respond to the innate charges of biomolecules adsorbed on the gate electrode by electrostatic interaction. The sensor surface was backfilled with sulfobetaine3-undecanethiol as an anti-fouling agent that prevents nonspecific adsorption of any protein in sample solutions. By designing such an interface, an electrical signal as a result of the specific adsorption of thrombin can be expected. Moreover, a DNA aptamer and FET are an ideal combination because the small size of the aptamer as a short oligonucleotide can capture the target in the proximity of the sensor surface, which is typically within the electrical double layer (a few nanometers in thickness). Goda and Miyahara also found that optimizing

Table 1

Comparison of each feature between natural and artificial receptors.

\begin{tabular}{lccccccc}
\hline Entry & Category & Sensitivity & Selectivity & Applicability & Stability & Durability & Quality \\
\hline Enzyme & Natural & Moderate & High & Wide & Low & Low & Diverse \\
Antibody & Natural & High & High & Wide & Low & Low & Diverse \\
$\begin{array}{l}\text { Aptamer } \\
\text { Biomimetic }\end{array}$ & Artificial & Moderate & High & Moderate & Moderate & High & Equal \\
receptor & Artificial & Moderate & High & Narrow & High & High & Equal \\
\hline
\end{tabular}




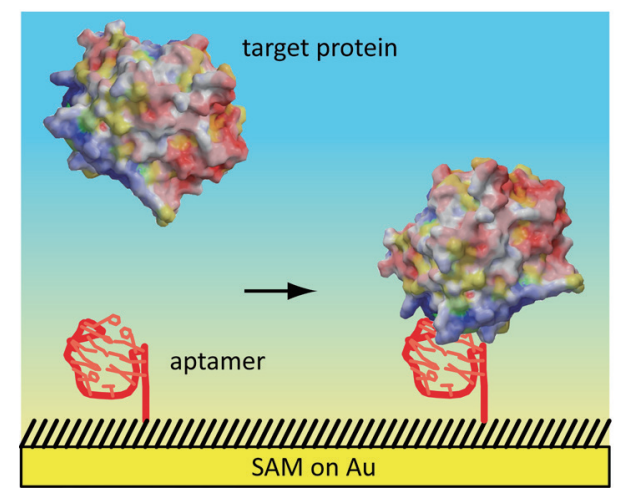

Fig. 2. (Color online) Biomarker thrombin was detected using a surface-immobilized DNA aptamer on an extended gold gate FET in a label-free and reagent-free manner. Reproduced with permission from Goda and Miyahara. ${ }^{(11)}$ Copyright (C) 2013, Elsevier B. V.

the nanoscale lateral distance between the surface-immobilized aptamers is essential to obtain their full binding performances. ${ }^{(11)}$ The biosensor was able to quantify thrombin with a dynamic range of 13.4-1300 $\mathrm{nM}$ and a limit of detection of $6.7 \mathrm{nM}$. The functional interface allowed the label-free measurement of thrombin in realistic samples that contained interfering serum components. DNA-aptamer-functionalized FET sensors may be applied to other targets; the lysozyme concentration was successfully determined by introducing the anti-lysozyme aptamer on the sensor surface instead of the anti-thrombin aptamer. ${ }^{(11)}$

One drawback of using a DNA aptamer for biosensing applications is the low binding affinity as compared with the antibody (Table 1). A reason for the superior binding affinity of antibodies to the target is a cooperative binding mechanism. Namely, antibodies can capture targets via multiple binding contacts. To overcome this issue, a functional interface that contains dual DNA aptamers for a single target protein was developed (Fig. 3). ${ }^{(12)}$ Dual aptamer ligands with serial or parallel connection to the sensor surface can squeeze the target thrombin on a gold electrode, thereby improving the apparent binding affinity. The study revealed that the arrangement of dual aptamers at the nanoscale on the sensor surface is essential to realize multiple binding to the target. Target-selective capturing with enhanced binding affinity was achieved by optimizing the linker and spacer lengths in the aptamer ligand molecules. It is feasible to use several aptamers that can bind to different epitopes in the target. This is because several candidate aptamers are found at one time during the process of selecting an aptamer sequence from random sequences, called the systematic evolution of ligands by exponential enrichment (SELEX). ${ }^{(13)}$ A strategy of molecular imprinting would help improve the efficacy of dual aptamer arrangements at the nanoscale on the sensor surface.

\section{Molecular Switch for ATP Detection}

In recent years, DNA-based nanoarchitectures have attracted widespread attention owing to their nanostructure-related functions such as molecular switches or nanomachines. The nanostructure is designed and built through Watson-Crick base-pairing. These mild intermolecular bindings based on hydrogen bonding allow structural transition induced by 


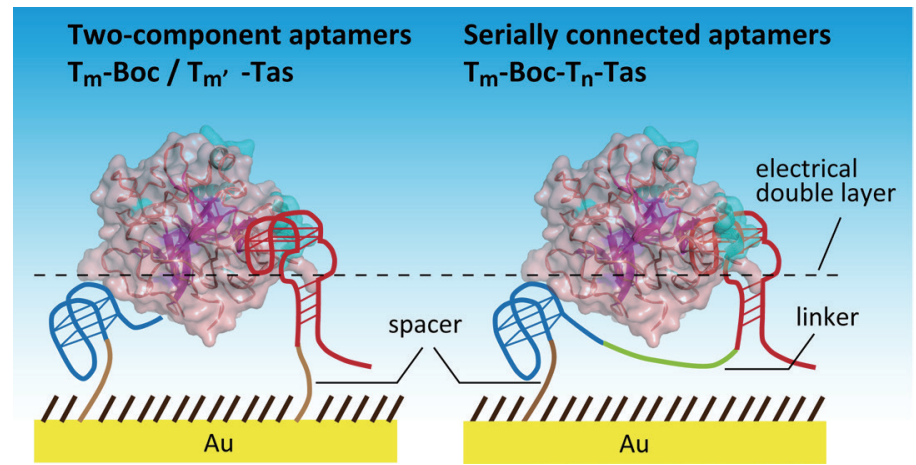

Fig. 3. (Color online) Thrombin recognition through multiple binding contacts improved the binding affinity. Spacer and linker lengths in the dual aptamers were a critical parameter. Adapted with permission from Goda et al. ${ }^{(12)}$ Copyright (C) 2015, Elsevier B. V.

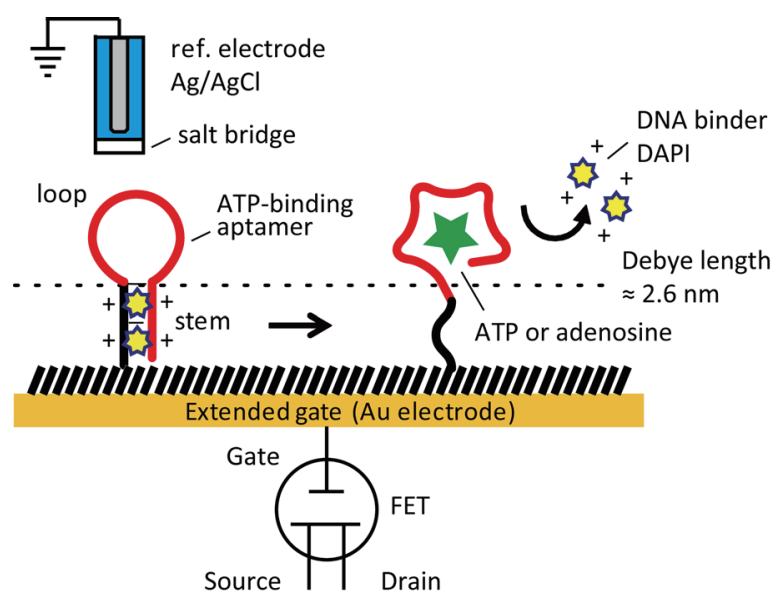

Fig. 4. (Color online) An electrical signal was transduced by conformational switching of the hairpin aptamer due to the release of the cationic DNA binder upon complexation with the target (ATP or adenosine) on an extended FET gate. Adapted with permission from Goda and Miyahara. ${ }^{(14)}$ Copyright ${ }^{C}$ 2011, Elsevier B. V.

physicochemical stimuli. Such nanostructural alteration can be transduced into a signal for biosensing applications. The features of DNA molecular switches spurred researchers to apply them to biosensing systems. A closed-loop hairpin DNA that contains an aptamer sequence against adenosine/adenosine triphosphate (ATP) has been immobilized on an extended gold gate FET (Fig. 4). ${ }^{(14)}$ The stem part of the hairpin can release a preloaded double-stranded DNA groove binder with positive charges to the solution phase. The structural transition was confirmed by total-internal-reflection fluorescence imaging. The release is accompanied by the structural transition to an open-loop conformation through the affinity of the hairpin aptamer to the target. The stem length needs to be optimized because it determines the kinetic barrier for the structural transition. ${ }^{(15)}$ The FET signal mainly depends on the release of the cationic DNA binder from the sensor surface. In other words, the mechanism enables the label-free detection of electrically neutral adenosine. The transduction mechanism by molecular switches may break the traditional concept that FET biosensors are limited to detecting charged species or ions. 


\section{Engineering Phospholipid for CRP Detection}

Many biomolecular recognition events take place on the cell surface to maintain homeostasis via cellular communications. The eukaryotic cell membrane is made of a self-assembly of phospholipid bilayers with a thickness of 6-10 nm. Ligands, receptors, and channels are embedded on the cell membrane and responsible for specific recognition. Also, different types and components of lipids are essential for the cell conditions. For example, phosphatidylserine is enriched in the outer membrane during apoptosis. ${ }^{(16)}$ This is the so-called eat-me signal that is accompanied by the recognition of immune cells for clearance. Moreover, the cell microenvironment influences the biorecognition event on the cell surface. C-reactive protein (CRP) is activated by the increase in calcium ion or proton levels in surrounding solutions. CRP is an acute-phase reactant during inflammation and infection. ${ }^{(17)}$ Its systemic level sharply increases by up to 1000-fold within $24 \mathrm{~h}$. Changes in the ionic microenvironment in damaged tissue may be involved in the enhanced binding affinity of circulating CRP to the cell surface. Mimicking such a dynamic molecular recognition system on the cell surface may lead to the development of a new biointerface that can interact with biological systems. First, the mechanism of the calcium- and $\mathrm{pH}$-dependent enhancement of CRP recognition on the cell surface was mimicked for label-free biosensing applications (Fig. 5). ${ }^{(18)}$ A zwitterionic phospholipid headgroup was introduced to a custom methacrylate polymer and the polymer was chemically immobilized on an amine-functionalized gold surface via the condensation reaction. The surface modification was characterized by surface plasmon resonance analysis. The polymer enabled the development of a biomembrane-mimetic interface on the surface of a label-free biosensor with improved robustness as compared with self-assembled lipid bilayers. ${ }^{(19)}$ The label-free biosensor enabled CRP detection of as low as $\sim 10 \mathrm{nM}$. The biomimetic surface helps elucidate the molecular dynamics. ${ }^{(20-22)}$ The binding was only mediated by calcium ions and not by other physiological cations. The binding constant coincided with the change in the systemic level of CRP in the acute phase. The biomembrane-mimetic surface was able to resist nonspecific adsorption of other proteins. This enabled CRP sensing in realistic serum samples. ${ }^{(23)}$

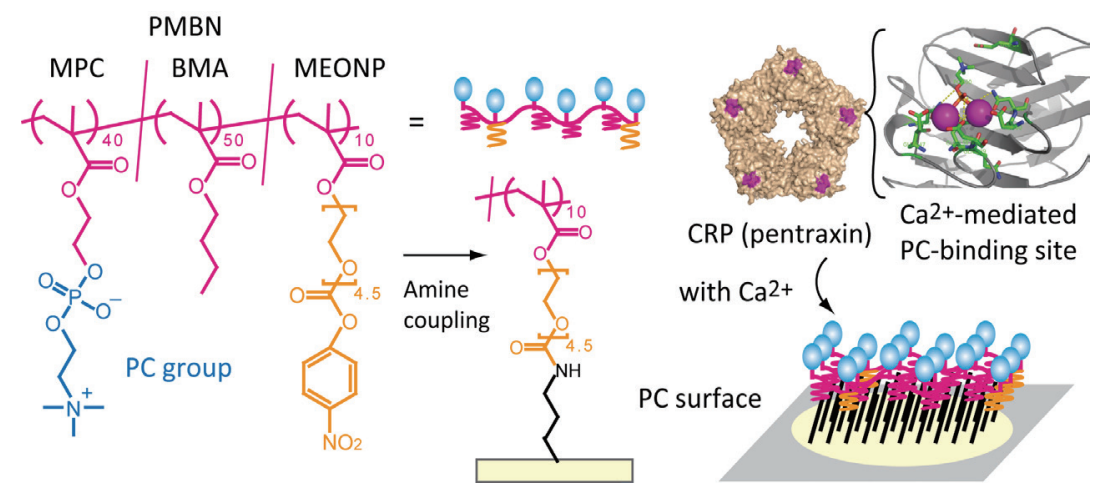

Fig. 5. (Color online) Covalent deposition of a custom biomimetic polymer onto a sensor surface was used to construct a robust biomembrane-mimetic surface on a label-free sensor. The engineered phospholipid surface revealed the calcium-mediated binding kinetics of CRP. Reproduced under the CC BY-NC-ND 3.0 license from Ref. 18. Copyright (C) 2014, The Authors, Published by WILEY-VCH Verlag GmbH \& Co. KGaA, Weinheim. 
CRP recognition capability was also imparted to a conducting polymer for label-free electrical biosensing (Fig. 6). ${ }^{(24)}$ Conducting polymers possess electronic and ionic conductivity in a doped state. In comparison with metallic and inorganic conductors, organic electronic materials are loosely assembled macromolecular clusters. As a result, the mass transfer of hydrated ions into the bulk phase of conducting polymer facilitates ionic current. The interfacial ionic mobilities realize low-impedance interfaces with biological systems. The mass productivity and workability of the conducting polymer with the aid of printing technologies open up a new concept. The introduction of ligand molecules into a conducting polymer is essential for label-free biosensing. To this end, the zwitterionic phospholipid headgroup was introduced to the side chain of a conducting polymer. Quantitative electrochemical signals were obtained due to calcium-dependent CRP binding on the surface of functional conducting polymers. The dynamic range was $10-160 \mathrm{nM}$ with a limit of detection of $37 \mathrm{nM}$. These values cover physiologically relevant CRP levels in the acute phase. The biosensor was regenerated by removing surface-bound CRP by treating with detergents.

\section{Engineering Oligosaccharide for Influenza Virus Detection}

Seasonal influenza epidemics cause severe damage to our society. Early diagnosis of infection is a crucial requirement for preventing the spread of the viruses because the current antiviral medication is valid within $48 \mathrm{~h}$ after the infection. Conventional methods rely on the detection of viral genes after amplification, which is time-consuming and expensive. Immunochromatographic tests are feasible for a simple inspection, but they are not sensitive. Moreover, the use of an antibody has a risk of false-negative results for the mutagenic influenza virus. A spike protein on the virus envelope plays an essential role in infection. The spike protein hemagglutinin selectively recognizes a specific oligosaccharide expressed on the cell surface for invasion. On the basis of this lectin-sugar specificity, we chemically introduced the specific oligosaccharide to the side chain of a functional conducting polymer for influenza virus detection (Fig. 7). ${ }^{(25,26)}$ Oligosaccharides show better stability and robustness than antibodies.

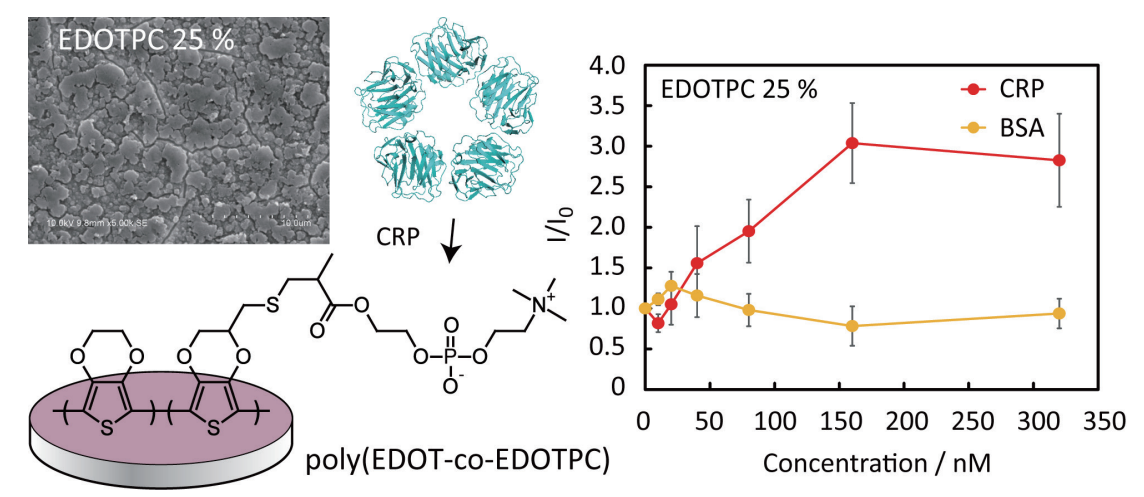

Fig. 6. (Color online) A phospholipid-headgroup-functionalized conducting polymer was deposited on an electrode for label-free electrochemical CRP biosensing. The electrochemical signal specifically increased with $\mathrm{CRP}$ concentration. Data are shown as mean $\pm \mathrm{SD}(n=3)$. Reproduced with permission from Goda et al. ${ }^{(24)}$ Copyright (C) 2015, American Chemical Society. 

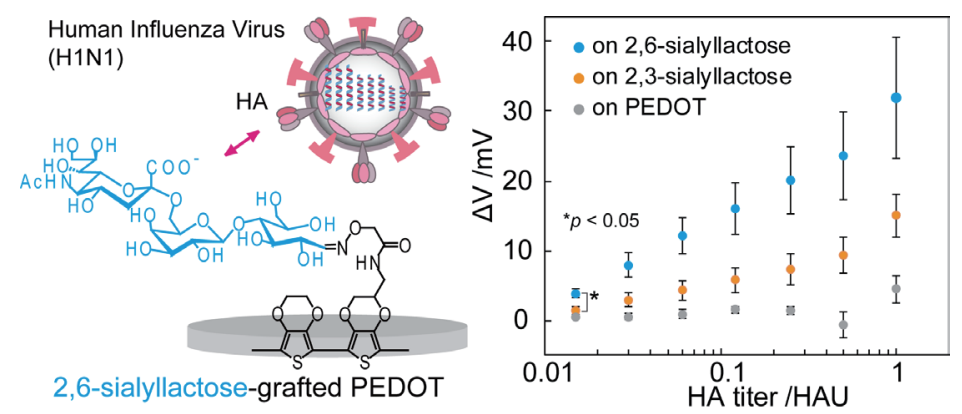

Fig. 7. (Color online) An oligosaccharide-modified conducting polymer realized specific electrical detection of the human influenza virus. The potential shift $(\Delta V)$ increased with the influenza virus concentration. Data are displayed as mean $\pm \mathrm{SD}(n=4) .{ }^{*} p<0.05$. Reproduced with permission from Hai et al. ${ }^{(25)}$ Copyright (C) 2017, American Chemical Society.

The biomimetic receptor can distinguish the human influenza virus from other influenza viruses. Moreover, a surface modified with an oligosaccharide as a hydrophilic compound effectively resists the nonspecific adsorption of biomacromolecules. Specific recognition of the virus was detected using a mass sensor a called quartz crystal microbalance (QCM) under wet conditions. The limit of detection was about two orders of magnitude better than that of commercial immunochromatographic tests. Moreover, the virus recognition generated electrical signals without the need for labeling because innate charges of spike proteins on the virus envelope or capacitive changes alter the conductivity of the conjugated main chain at the interface. The sensitivity in electrical measurements was comparable to that of the QCM sensor. Owing to the processability, workability, and mass productivity, the sugar-functionalized conducting polymer could be used in point-of-care testing.

\section{Conclusions}

Label-free biosensing is cost-efficient and straightforward. It can exclude any possible artifacts brought out by labeling processes or tagged molecules. The key requirements for labelfree biosensing are how to realize specific biorecognition and how to fit the signal transduction mechanisms at the sensor/solution interface. In this review, the design and fabrication of biointerfaces are described with some examples of electrical and electrochemical sensing. Aptamers are engineered biorecognition elements with the features of durability, equal quality, and availability. An artificial receptor can specifically interact with a target compound from impure samples. Moreover, designer sequences realize cooperative binding and target-induced structural alteration. Mimicking biorecognition via phospholipids or sugars on cell surfaces can be used to inspect the binding mechanisms of an acute-phase reactant in our bodies at the molecular level. It also provides an efficient methodology for distinguishing human-pathogenic viruses from others. Preventing nonspecific binding of impurities on a sensor surface is realized by introducing anti-fouling zwitterions such as biomembranes. These core techniques for developing biointerfaces are essential for performing point-of-care testing and developing wearable biosensing devices. 


\section{Acknowledgments}

This work was supported by a JSPS Grant-in-Aid for Young Scientists (B) (23750079), the JSPS Program on Strategic Young Researcher Overseas Visits for Accelerating Brain Circulation (G2303), JSPS Grants-in-Aid for Scientific Research on Innovative Area (24107507, 26107705), the Futaba Electronics Foundation, and the Asahi Glass Foundation.

\section{References}

1 N. Rifai, M. A. Gillette, and S. A. Carr: Nat. Biotechnol. 24 (2006) 971. https://doi.org/10.1038/nbt1235

2 S. Ray, G. Mehta, and S. Srivastava: Proteomics 10 (2010) 731. https://doi.org/10.1002/pmic. 200900458

3 M. A. Cooper: Anal. Bioanal. Chem. 377 (2003) 834. https://doi.org/10.1007/s00216-003-2111-y

4 J. Kim, A. S. Campbell, B. E. F. de Avila, and J. Wang: Nat. Biotechnol. 37 (2019) 389. https://doi.org/10.1038/ s41587-019-0045-y

5 T. Goda, K. Masuno, J. Nishida, N. Kosaka, T. Ochiya, A. Matsumoto, and Y. Miyahara: Chem. Commun. 48 (2012) 11942. https://doi.org/10.1039/c2cc36111f

6 E. Kim, M. D. Baaske, and F. Vollmer: Lab Chip 17 (2017) 1190. https://doi.org/10.1039/c6lc01595f

7 S. Erbas-Cakmak, S. Kolemen, A. C. Sedgwick, T. Gunnlaugsson, T. D. James, J. Yoon, and E. U. Akkaya: Chem. Soc. Rev. 47 (2018) 2228. https://doi.org/10.1039/c7cs00491e

8 K. Ishihara: J. Biomed. Mater. Res. A 107 (2019) 933. https://doi.org/10.1002/jbm.a.36635

9 K. Ishihara, M. W. Mu, T. Konno, Y. Inoue, and K. Fukazawa: J. Biomat. Sci. Polym. Edn. 28 (2017) 884. https://doi.org/10.1080/09205063.2017.1298278

10 J. W. Liu, Z. H. Cao, and Y. Lu: Chem. Rev. 109 (2009) 1948. https://doi.org/10.1021/cr030183i

11 T. Goda and Y. Miyahara: Biosens. Bioelectron. 45 (2013) 89. https://doi.org/10.1016/j.bios.2013.01.053

12 T. Goda, D. Higashi, A. Matsumoto, T. Hoshi, T. Sawaguchi, and Y. Miyahara: Biosens. Bioelectron. 73 (2015) 174. https://doi.org/10.1016/j.bios.2015.05.067

13 R. Stoltenburg, C. Reinemann, and B. Strehlitz: Biomol. Eng. 24 (2007) 381. https://doi.org/10.1016/ j.bioeng.2007.06.001

14 T. Goda and Y. Miyahara: Biosens. Bioelectron. 32 (2012) 244. https://doi.org/10.1016/j.bios.2011.12.022

15 T. Goda and Y. Miyahara: Biosens. Bioelectron. 26 (2011) 3949. https://doi.org/10.1016/j.bios.2011.02.041

16 J. Savill, I. Dransfield, C. Gregory, and C. Haslett: Nat. Rev. Immunol. 2 (2002) 965. https://doi.org/10.1038/ $\underline{\text { nri957 }}$

17 M. B. Pepys and G. M. Hirschfield: J. Clin. Invest. 111 (2003) 1805. https://doi.org/10.1172/Jci200318921

18 T. Goda, P. Kjall, K. Ishihara, A. Richter-Dahlfors, and Y. Miyahara: Adv. Healthc. Mater. 3 (2014) 1733. https://doi.org/10.1002/adhm.201300625

19 T. Goda, K. Ishihara, and Y. Miyahara: J. Appl. Polym. Sci. 132 (2015) 41766. https://doi.org/10.1002/app.41766

20 T. Goda and Y. Miyahara: Acta Biomater. 48 (2017) 206. https://doi.org/10.1016/j.actbio.2016.10.043

21 T. Goda and Y. Miyahara: Colloids Surf. B 161 (2018) 662. https://doi.org/10.1016/j.colsurfb.2017.11.036

22 T. Goda and Y. Miyahara: Acta Biomater. 40 (2016) 46. https://doi.org/10.1016/j.actbio.2016.02.008

23 T. Shimada, T. Yasui, A. Yokoyama, T. Goda, M. Hara, T. Yanagida, N. Kaji, M. Kanai, K. Nagashima, Y. Miyahara, T. Kawai, and Y. Baba: Lab Chip 18 (2018) 3225. https://doi.org/10.1039/c8lc00438b

24 T. Goda, M. Toya, A. Matsumoto, and Y. Miyahara: ACS Appl. Mater. Interfaces 7 (2015) 27440. https://doi. org/10.1021/acsami.5b09325

25 W. F. Hai, T. Goda, H. Takeuchi, S. Yamaoka, Y. Horiguchi, A. Matsumoto, and Y. Miyahara: ACS Appl. Mater. Interfaces 9 (2017) 14162. https://doi.org/10.1021/acsami.7b02523

26 W. F. Hai, T. Goda, H. Takeuchi, S. Yamaoka, Y. Horiguchi, A. Matsumoto, and Y. Miyahara: Sens. Actuators, B 260 (2018) 635. https://doi.org/10.1016/j.snb.2018.01.081 


\section{About the Author}

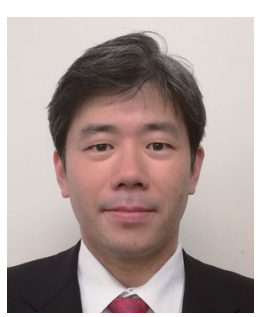

Tatsuro Goda received his B.S. degree from Kyoto University in 2003 and his M.S. and Ph.D. degrees from the University of Tokyo in 2005 and 2008, respectively. After a few years of postdoctoral work, he became an assistant professor at Tokyo Medical and Dental University in 2010, where he worked until 2019. Since 2020, he has been an associate professor at Toyo University. His research interests are in biomimetic engineering, biosensing, and bioelectronics. (goda@toyo.jp) 